\title{
A heuristic method for transmission design
}

\author{
Viktor Ivanov ${ }^{1, *}$, Lubomir Dimitrov ${ }^{2}$, Svitlana Ivanova $^{3}$, and Galyna Naleva $^{4}$ \\ ${ }^{1}$ Odessa National Polytechnic University, Ukraine \\ ${ }^{2}$ Technical University of Sofia, Bulgaria \\ ${ }^{3}$ South Ukrainian National Pedagogical University named after K. D. Ushynsky, Ukraine \\ ${ }^{4}$ National University "Odesa Maritime Academy"
}

\begin{abstract}
The application of heuristic methods in the design of transmissions is investigated. The structure of heuristic methods is revealed and the heuristic techniques that compose them are established. These techniques are combined into one generalized method, which allows replacing all the considered methods. A heuristic method specially allotted for the design of transmission is also developed. This method contains, among other things, the following heuristic techniques. Search for all possible variants of elements location corresponding to one of the same graph. Introduction nodes to the graph that corresponding certain function, for the implementation of which a node can be added to the design or vice versa, a node can be removed from the design, and the function it has implemented will be implemented by other elements. The advantages of a heuristic search on the example of designing a double row thrust bearing are evidenced. The bearing contains a node through which the speed of the intermediate ring is always equal to half the speed of the shaft. Due to this, the distance that the rolling element path is reduced, and the bearing has greater durability.
\end{abstract}

\section{Introduction}

The main direction of research in power transmissions is the development of calculation methods used software packages of computer-aided design. One of the consequences of this approach is the maximum possible complication of design schemes, with the aim of using all the possibilities inherent in software packages. This approach does not correspond to the real problems of creating a new transmissions design and often such research are focused on secondary tasks. The inability to achieve essential progress in this way is evident from the fact that for the past fifty years, standard methods for calculating gears and bearings have not significantly changed.

The most difficult task of transmissions design - the creation in principle new designs is not given enough attention. Heuristic methods are not part of the software packages of computer-aided design. Software packages for solving heuristic problems are very few. These software packages are universal and can be used in different areas of technology. There are no elaborations related to the heuristic methods suitable for solving problems directly related to the transmissions design.

There is a widespread opinion that for such a simple object as a transmission the use of heuristic methods is not advisable. But every year hundreds of new designs of bearings, gear wheels and couplings are invented. The inventors need a heuristic method to facilitate their research. A heuristic method that is directly intended for designing transmissions can be involved as part of software packages of computer-aided design.

\section{Literature review and problem statement}

The most widely whispered heuristic methods are based on the design representation in the form of a matrix or graph. Matrices are also used to present information about design elements in a form suitable for computer processing. The original idea of the morphological maps method is to use matrices to generate all possible combinations of elements. This method allows, for example, comparing the functions of machine and unit which realized this functions [1]. A particular, but very effective way of constructing the matrix is to use the design features as columns, and the types of gearboxes as rows [2].

A DSM (design structure matrix) matrix is compiled in order to integrally assess the allowable changes with the help of which the presence or absence of an interrelation between product nodes is established. Based on the found relationships, the elements are rearranging (change the position of the elements in the rows and columns) so that the interrelated elements form a cluster [3]. The interaction matrix method is aimed at identifying the interrelations between the structural elements and their systematization. The application of the interaction matrix method requires the following steps: to define the concepts of «element» and «interrelation»; to create a matrix, in which each element can be matched with any other; on the basis of objective data, to determine whether there is a relationship between each pair of elements [4]. The degree of influence of one element on another can be estimated in

\footnotetext{
Corresponding author: ivv@opu.ua
} 
the matrix. Usually for this purpose use a certain rating scale.

The TRIZ method provides for the formation of a matrix in which nodes and elements of the product are located horizontally, and functions or parameters are located vertically. The contents of the matrix cells are the list of techniques that can be used to eliminate technical contradictions [5].

A matrix diagram (quality table) is a tool that allows you to determine the presence and importance of interrelations between the elements, functions or characteristics of an object. It is a table that includes the elements between which you need to establish a relationship. The first row and column contains the elements to be examined, the other cells contain symbols or numbers indicating the presence and strength of relationship. It is suggested that the columns should be the main causes of the problem, and the lines - possible means of eliminating these causes [6].

The method of analysing interconnected decision areas (AIDA) is aimed at identifying and evaluating all compatible combinations of individual solutions to the problem. The application of the AIDA method requires the following stages: to identify several possible options in each decision area; to specify which options are incompatible and which can be combined with each other; to find the option that best suits the problem [7].

The interaction network method partially repeats the steps inherent in the interaction matrix method. But the analysis is based not on the construction of a matrix, but on the construction of a graph in which - the vertices are elements, and the edges - the interrelation between the elements. Then you need to change the position of the vertices so as to minimize the number of intersections of edges and identify the network structure more clearly [8].

One way of the mechanism description in the form of a graph is based on the design method using signal flow graphs (SFG) [9]. In this method, the nodes are variables, and the edges are functional dependencies. The SFG method is based on the four following principles. Direction of flow between nodes is designated by arrows. To obtain the output signal, the input signal is multiplied by a weight factor of a given edge. The value of a signal in a node is the sum of all input signals. The value of a signal in a node is transmitted by all the edges that extend from it. The SFG method is widely used by other researchers, for example, to study power flows in planetary gears. The nodes are the gears, and the edges of the equation of moments [10].

Bond-graphs offer a great opportunity for analysis of mechanisms and electromechanical systems. The bondgraph for a metal V-belt CVT, in which edges of the graph are in conformity with angular velocities and moments, and nodes are mechanical parts (nodes) and electrical components, was developed [11]. It was noted that a bond-graph is a unique visual way of graphic analysis of power flow, the latter is important, not only for analysis, but also to search for new design solutions.

Some heuristic methods that are used for reverse engineering can be effective for designing transmissions. One of these methods is change impacts on complex product (CICP). An analysis of the changes, which are made in product design for the purpose of redesign, is carried out using a graph of weight coefficients. First, the product design is divided into nodes and identified the interrelation between the elements, then represents the structure in the form of a graph. After that, a matrix is formed in which the degree of impact the changes of one node to another are estimated on a scale: direct influence, indirect influence, inadmissible [12].

The change prediction method (CPM) consists of three steps: creating the product model; completing the dependency matrices and computing the predictive matrices. The basic structure of the model is an amalgamation of DSM matrices and risk matrices methods. Products are modelled as linked components, where interrelations consist of separate probability and impact terms. The evaluation of probability and impact on the components interrelations with using matrices is carried out. At the risk matrix the interrelations of the components design and the possible causes of failures are learning [13].

The reverse engineering and redesign methodology (RERM), which three phases consists: reverse engineering, modelling and analysis, upgrade, is developed [14]. An object is considered as a black box and the product's parameters are determined, its functions are established. The hypothesis is put forward regarding the physical principles that are used in the object and its design. Three alternative design variants of the product are creating. In the first case, the product parameters change, and in the second case the product features also change. In the third case, is developing a fundamentally new product design with using morphological map in which functions of the product are located horizontally, and possible options of design solutions are located vertically. Evaluation of product design quality is carried out using the quality table [14].

At the heuristic method for identifying modules for product architectures (IMPA) functions for which the product is intended are determined [15]. The product is considered as a black box and certain functions are compared with known nodes that can perform these functions. A heuristic method is used to determine the mechanical, electrical, hydraulic power flows that are necessary to implement the product functions. Then the search for unites is carried out, which is not enough to perform certain functions or that would perform these functions more efficiently than the existing architecture. The search with using morphological map and DSM matrix is carrying out. Unlike the method (RERM), flow analysis is performed using a flowchart [15].

The above heuristic methods are diverse; each involves a certain algorithm of action, including heuristic techniques and procedures performed in a certain sequence. To choose the most suitable method and to study its features of its application is not an easy task.

For recommendations on the use of heuristic methods, it is necessary to give an analysis of their structure. From each method, heuristic techniques that are used in it should be identified. The criterion for the selection of heuristic methods is the possibility of their use in the design of transmissions. The aim of the 
research is to create a heuristic method aimed at developing new transmission designs based on techniques, which are part of known heuristic methods.

\section{Structure of heuristic methods}

Different authors use the same set of heuristic techniques to solve problems in various fields of machinery. In some publications, the object was considered as a black box. Some researchers is used the morphological map or DSM matrix as a part of own heuristic method. It should be noted that the publications devoted to upgrade and redesign are based on heuristic methods to which special titles are given. These titles do not correspond to the commonly used terminology of heuristics. In fact, proposed methods are combination of widely known methods: morphological map, network of interactions, matrix diagram and SFG. As example such combination, at the search for the possible use of known transmissions in the form to the nodes of a CVT was performed using a morphological map [16]. To search for a new design, an interrelation graph was used. The final version of the design was obtained taking into account the analysis of power flows, and the evaluation of design options was performed using a matrix diagram [16].

The considered heuristic methods have many common features. The analysis showed that all methods, as the first stage, assume the separation of the system into elements. The elements are nodes (engine, CVT, gearbox, transmission, and operating mechanisms) or machine elements. Another stage is the establishment of interrelations between the elements using the constructed matrix or graph. Matrices and graphs are complementary ways of expressing the same system of interrelations. These two methods can be used simultaneously.

Widely known heuristic methods have many same techniques too. In DSM matrix, morphological maps and interaction network, usually only the fact of interrelation between product nodes is established. In the DSM matrix, AIDA and interaction network, identify of interrelated groups of elements. Most of them, as the stage have establishing the degree of influence between elements qualitatively or numerically (Table 1).

For this analyze, the definition of the heuristic method and the heuristic technique are given below. Heuristic method is a combination of heuristic techniques and procedures allowing intensifying and organizing the thinking. Heuristic technique is a procedure that requires the participation of a designer.

And also an axiom and theorem are formulated.

Axiom: The heuristic method must contain at least one heuristic technique.

Theorem: If two heuristic methods contain the same heuristic techniques and differ only in the procedures performed, then these are modifications of the same method.

The analyze a structure of the methods described above is made using definition of the heuristic method and the heuristic technique, also an axiom and theorem.
Table 1. Heuristic method and techniques.

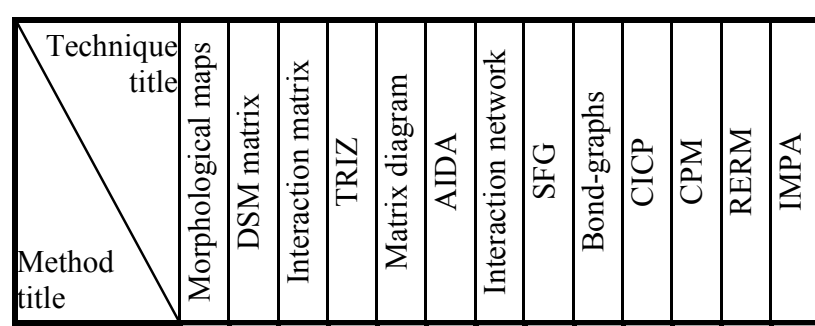

Selecting

elements

Establishing

a set of

functions or

function

parameters

Establishing

a set of

physical

principles

Establishing

elements

inter-

relations

Identifi-

cation of

interrelated

groups of

elements

Establishing

the degree

of influence

between

elements

qualitatively

or

numerically

Establishing

inter-

relations

between

elements

and

functions

performed

Establishing

the flow of

power, heat,

information

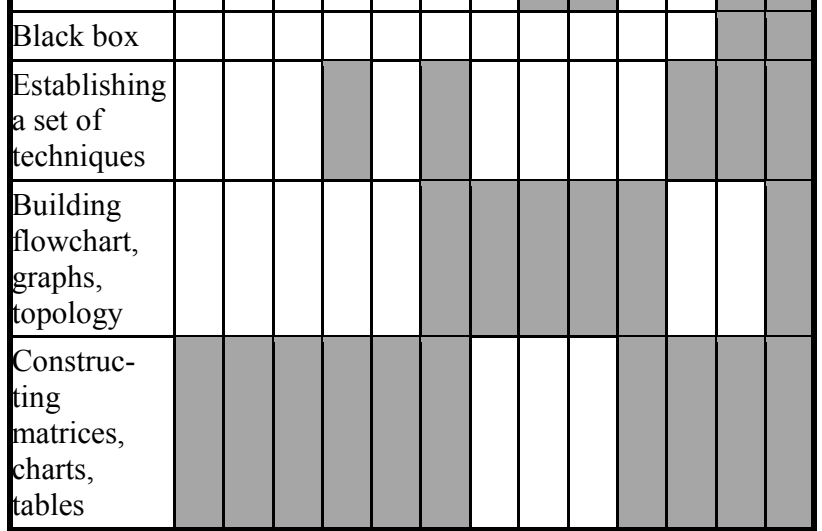


From each method, the heuristic technique that is used in it is selected. Instead of applying all the methods described, one method can be used: the generalized method, which includes all identified heuristic techniques.

The heuristic technique "Establishing a set of physical principles" is not relevant for the design of transmissions, since transmission is mechanical system. Heuristic technique of the "Black box" is more suitable for solving problems of redesign, and the technique «Establishing a set of techniques" is not relevant because we have formed the whole possible list of receptions.

The heuristic techniques "Selecting elements" and "Establishing elements interrelations" all methods are contained. In transmissions the technique "Selecting elements" very often means that a list of original machine elements are need constructed. Technique "Establishing elements interrelations" can mean only that machine elements which are part of node are need selected. Therefore, these techniques may not always be heuristic. We believe that this procedures of transmissions design.

Thus, the following techniques are included in the generalized method: establishing a set of functions or function parameters; identification of interrelated groups of elements; establishing the degree of influence between elements qualitatively or numerically; establishing interrelations between elements and functions performed; establishing the flow of power, heat, information; building flowchart, graphs, topology; constructing matrices, charts, tables.

\section{Development heuristic method}

For an example of the use of selected heuristic techniques, consider a search for a thrust bearing new design.

Using heuristic procedure - "Selecting elements". As elements, according to the type of bearing in the direction of perceived force, are types of bearings: radial, angular contact and thrust. Elements according to the type of bearing under the number of rows of rolling elements are: single row and double row.

Using heuristic technique - "Constructing matrices". In the cells of the matrix, it is indicated how an increase in the number of rows affects the durability of the bearings (Table 2).

Table 2. Morphological map (base - base durability, more increased durability)

\begin{tabular}{|c|c|c|}
\hline $\begin{array}{r}\text { Number of rows of rolling } \\
\text { elements }\end{array}$ & $\begin{array}{c}\text { Single } \\
\text { row }\end{array}$ & $\begin{array}{c}\text { Double } \\
\text { row }\end{array}$ \\
\hline Direction of perceived effort & base & more \\
\hline Radial & base & more \\
\hline Angular contact & base & base \\
\hline Thrust
\end{tabular}

Double row bearings, radial and angular contact, are used to increase durability, and double direction thrust bearings only for the ability to perceive axial force in both directions. The thrust bearing design, in which the addition of another row of rolling elements will improve durability, is the aim of heuristic search.

Using heuristic procedure - "Selecting elements". As elements accept $A_{0}$ - axis hole in the housing, $A_{1}$ - shaft axis, $D_{0}$ - fixed ring, $D_{1}$ - rotating ring, $D_{2}$ - intermediate ring, $R_{1}$ - rolling elements of the upper row, $R_{2}$ - rolling elements of the lower row. The bearing shaft is connected to the intermediate ring (Figure 1).

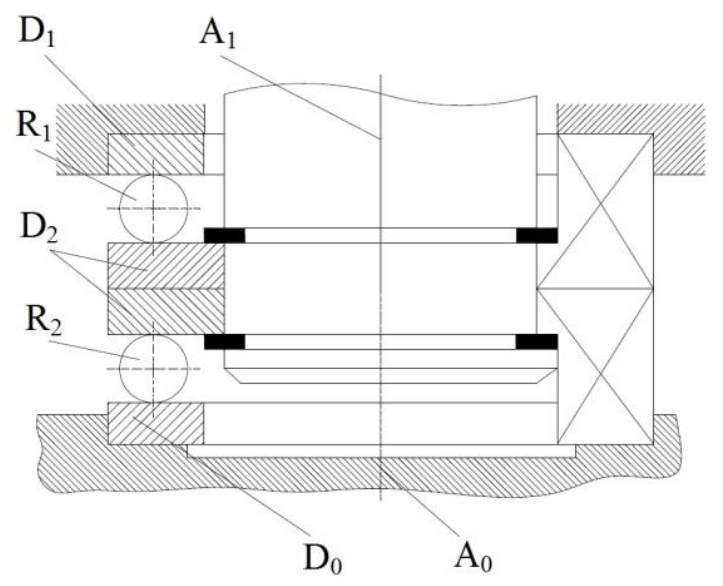

Fig. 1. Design of double row thrust bearing.

Using heuristic techniques - "Constructing graph". The graph constructed for two cases when the force is directed up (a) and down (b) (Figure 2). When the axial force of the upward load is perceived by the upper row of rolling elements, and then by the upper ring mounted in the housing. With the axial force directed downward, the load is perceived by the lower row of the rolling elements, and then by the lower ring mounted in the body.

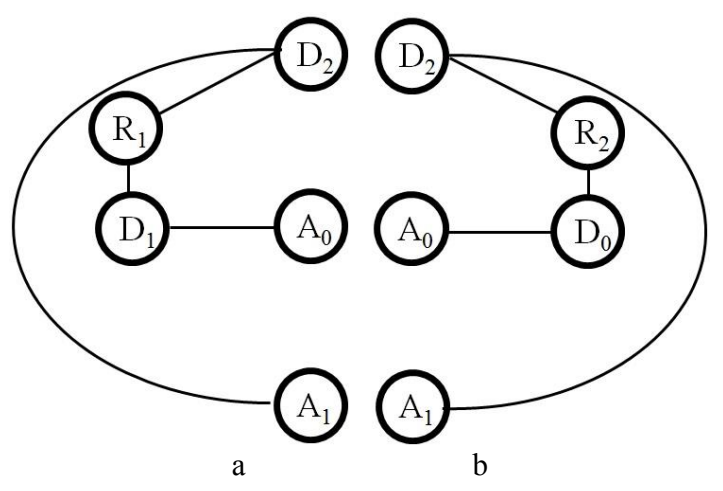

Fig. 2. Graph for force directed up (a) and down (b).

Using heuristic techniques - "Establishing a set of functions or function parameters". An analysis of the thrust bearing design shows that the double bearings are not used due to the fact that the rotational speed of the intermediate ring is unknown. It can be rotated at the same angular velocity as the upper ring, but may be fixed as the lower one, depending on the ratio of frictional forces in contact of balls with races. At the same time, this option of using an thrust bearing is promising, since if the intermediate ring rotated at an angular speed equal to half the angular velocity of the shaft, then each of the rows of the rolling elements 
would travel half the time for one revolution of the upper ring. That is, a new function should be added - ensuring an angular velocity equal to half the angular velocity of the shaft.

Using heuristic techniques - "Establishing interrelations between elements and functions performed". The outer and inner bearing rings provide a function the movement of centres of rolling elements in a circle. This function can be performed by other parts - a sleeve and a journal shaft with specially treated surfaces. The separator, on the one hand, is an element, on the other hand, it provides the function - the rolling elements must be located at the same distance. The element separator may not be, for example, in needle roller bearings, and the function - rolling elements must be located at the same distance is always present. To perform the function - providing angular velocity equal to half the angular velocity of the shaft, a particular unit must be added to the bearing design.

Using heuristic techniques - "Constructing graph".

In this way, a function can be introduced into the transmission graph in the form of a node, for the realization of which an element or a node can be added to the design. Or vice versa, the node of the graph is a element that can be removed from the design, and the function that it implemented will be implemented by other elements. The graph corresponding to the original bearing design is constructed, when the speed of the intermediate ring depends on the ratio of frictional forces in contact (Figure 3a). In the bearing graph, we introduce a node $\mathrm{J}$, which provides the function - the angular velocity is equal to half the angular velocity of the shaft (Figure 3b).

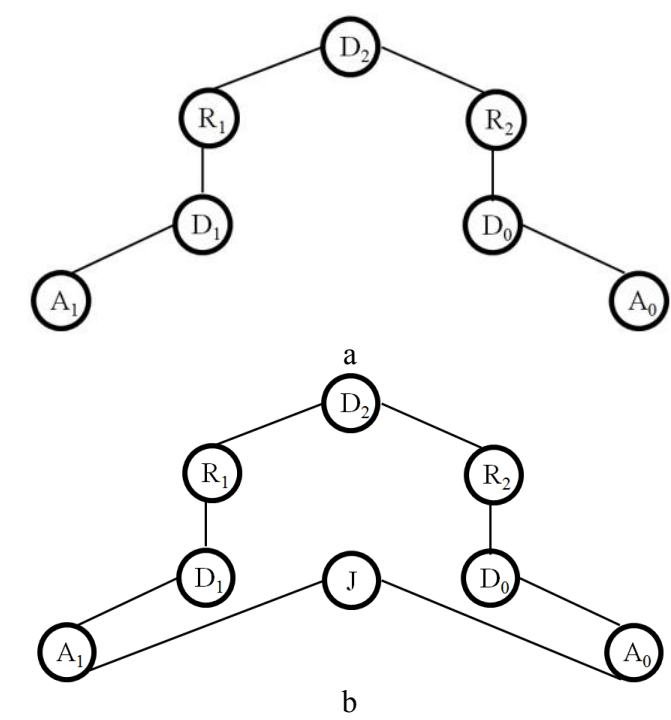

Fig. 3. Graph of standard bearing (a), graph of bearing with speed control node (b).

In a bearing design, the node $\mathrm{J}$ is implemented as a differential bevel gear train. The angular velocity of the upper sun gear is equal to the angular velocity of the shaft, the angular velocity of the lower sun gear is zero and the angular velocity of the planet carrier is equal to half the angular velocity of the shaft (Figure 4a). Denote additionally: $Z_{1}$ is the upper sun gear, $Z_{0}$ is the lower sun gear, $Z_{2}$ and $Z_{2}$ are planets which rotate on the $J_{0}$ axis (Figure $4 b$ ).
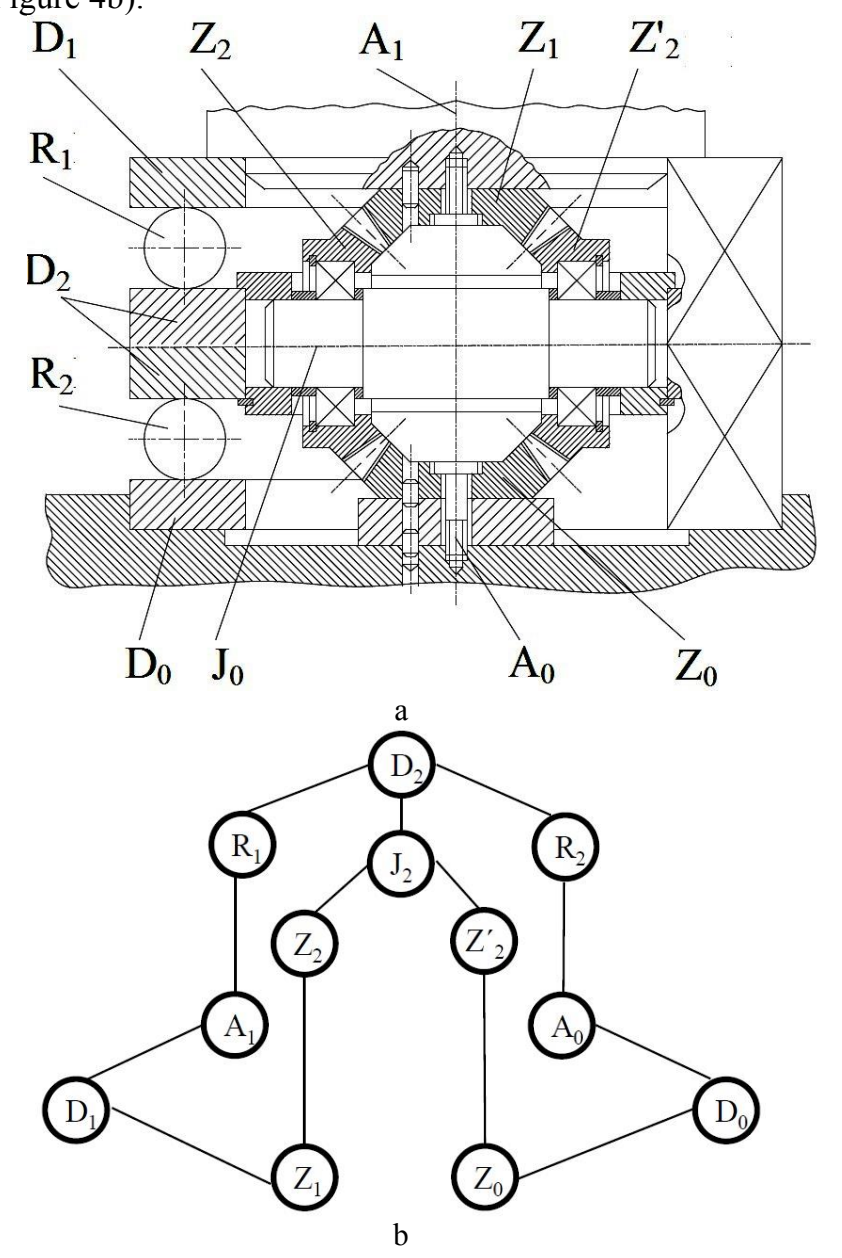

Fig. 4. Proposed design of double row thrust bearing (a), graph of proposed bearing (b).

Such a bearing design ensures the equality of a path that passes the rolling elements the upper and lower rows. They pass a distance is two times less of a distance which rolling elements of a single row thrust bearing are passed. Thus, the durability of the proposed rolling bearing is higher than the single row bearing and, in some cases, it can be used instead of hydrodynamic slider bearings.

\section{Conclusion}

Heuristic methods used in mechanical engineering were selected. The analysis of their structure is carried out. Heuristic techniques, which is components these methods were installed. A generalized heuristic method including selected heuristic techniques has been created. To solve the problems inherent in mechanical engineering, a new heuristic method has been developed, which includes four heuristic techniques:

- reducing the number of nodes in the graph while simplifying the design or for cutting off not essential elements;

- change the location of the graph vertices then to search necessary changes in the design which corresponds to the new location of the vertices; 
- search for all possible variants of elements location corresponding to one of the same graph;

- introduction nodes to the graph that corresponding certain function, for the implementation of which a element or node can be added to the design or vice versa, a element or node can be removed from the design, and the function it has implemented will be implemented by other elements.

The design of the double row thrust bearing is developed using the proposed heuristic method.

\section{References}

1. M. Fargnoli, E. Rovida, R. Troisi In: Proc. ICAD (2006)

2. V. Ivanov, G. Urum, S. Ivanova, G. Naleva East.Eur. J. of Enterprise Technolog., 4, 1, 11-17 (2017)

3. A. Yassine Urbana 51, 9 1-17 (2004)

4. M. Jovanovski, C. Popovska, K. Donevska, I. Peshevski In 11th Symposium, WMHE, Ohrid (2009)

5. C. Pokhrel, C. Cruz, Y. Ramirez, A.Kraslawski Chemical Engineering Research and Design, 103 (2015)

6. E. Vezzetti, F. Marcolin, A.L. Guerra Intern. J. of Quality \& Reliability Manag. 33, 2 (2016)

7. A. Weas, M. Campbell AI EDAM, 18, 3, 227-243 (2004)

8. P. Battaglia, R. Pascanu, M. Lai, D.J. Rezende In: Advances in neural inform. proces. syst., 4502-4510 (2016)

9. R. Ma, K.C. Gupta Journal of Mech. Des., 116, 1, 326-331 (1994)

10. K. Arnaudov, D. Karaivanov Transactions of FAMENA, 37, 1, 49-61 (2013).

11. J. Deur, V. Ivanovic, F. Assadian, M. Kuang, H.E. Tseng, D. Hrovat, In: Proc. of the 7th Vienna Intern. Conf. on Math. Model (2012)

12. H. Cheng, X. Chu J. of Intelligent Manufacturing, 23, 4, 1419-1431 (2012)

13. P.J. Clarkson, C. Simons, C. Eckert J. of Mech. Des. 126, 5, 788-797 (2004)

14. K. N. Otto, K. L. Wood Research in Eng. Des., 10, 4, 226-243 (1998)

15. R.B. Stone, K.L. Wood, R.H. Crawford Des. studies, 21, 1, 5-31 (2000)

16. V. Ivanov, G. Urum, S. Ivanova, M. Volkova, East.Eur. J. of Enterprise Technolog., 1, 3, 43-50 (2018) 